\title{
ON A CONSTRUCTIVE DEFINITION OF THE RESTRICTED DENJOY INTEGRAL
}

\author{
BY \\ DONALD W. SOLOMON
}

1. Introduction. Let $I=[a, b]$ and $F$ be a function of the closed subintervals of $I$. One says that $F$ has Burkill integral $\int_{J} F$ over the closed interval $J \subseteq I$ if

$$
\int_{J} F=\lim _{P \in \mathscr{P}:|P| \rightarrow 0} \sum_{I^{\prime} \in P} F\left(I^{\prime}\right)
$$

where $\mathscr{P}$ is the set of partitions of $J$ and $|P|$ is the norm of $P$. The Burkill integral has been employed in a more general setting [1] to give a descriptive definition of the restricted Denjoy integral of point functions $f$. In this paper we show how this integral can be used to give a constructive definition of the restricted Denjoy integral and compare the classical construction with ours. We adopt the convention that $I$ and $J$, with or without subscripts or superscripts, always denote a closed interval.

Before we begin our discussion, let us recall the classical constructive definition ([2], 255-259) of the restricted Denjoy integral.

Let $T$ be a real-valued function whose domain, $\operatorname{dom} T$, is a set of ordered pairs $\{(f, J)\}$, where $f$ is a real-valued point function defined on $J$. The set

$$
\{f:(f, J) \in \operatorname{dom} T\}
$$

will be denoted by dom, $T$.

$T$ is called an integral if

(i) $f \in \operatorname{dom}_{J} T$ implies $f \in \operatorname{dom}_{J}, T$ for all closed subintervals $J^{\prime} \subseteq J$, and $T\left(f, J^{\prime}\right)$ is an additive, continuous function of $J^{\prime}$;

(ii) if $f \in \operatorname{dom}_{I_{i}} T, i=1,2$, where $I_{1}$ and $I_{2}$ are abutting, then $f \in \operatorname{dom}_{I_{1} \cup I_{2}} T$;

(iii) if $f \equiv 0$ on $I$, then $f \in \operatorname{dom}_{I} T$ and $T(f, I)=0$.

One says that $f$ is $T$-integrable on $I$ if $(f, I) \in \operatorname{dom} T$.

Two integrals $T_{1}$ and $T_{2}$ are compatible if $T_{1}\left(f, I^{\prime}\right)=T_{2}\left(f, I^{\prime}\right)$ whenever they both exist. We say $T_{1} \subseteq T_{2}$ if $T_{1}$ and $T_{2}$ are compatible and $\operatorname{dom} T_{1} \subseteq \operatorname{dom} T_{2}$.

Given a function $f$ defined on $I^{\prime} \subseteq I$ and an integral $T$, one says that a point $x \in I^{\prime}$ is a $T$-singular point of $f$ in $I^{\prime}$ if there exists $\left\{I_{n}\right\}$, with $I_{n} \subseteq I^{\prime},\left|I_{n}\right| \rightarrow 0, x \in I_{n}$, such that $\left(f, I_{n}\right) \notin \operatorname{dom} T$.

If $\mathscr{S}$ is the set of $T$-singular points in $I$, clearly $\mathscr{S}$ is closed and $\left(f, I^{\prime}\right) \in \operatorname{dom} T$ for all $I^{\prime} \subseteq I$ such that $I^{\prime} \cap \mathscr{S}=\varnothing$.

Received by the editors October 4, 1966. 
Let $T$ be an integral. One defines $\operatorname{dom}_{I} T^{C}$ by the following conditions:

$\left(\mathrm{c}_{1}\right) \mathscr{S} \cap I$ is finite or void;

$\left(\mathrm{c}_{2}\right)$ there exists a continuous, additive $F$ such that $F\left(I^{\prime}\right)=T\left(f, I^{\prime}\right)$ whenever $\left(f, I^{\prime}\right) \in \operatorname{dom} T, I^{\prime} \subseteq I$.

Define $T^{C}(f, I)=F(I)$. It is clear that $T^{C}$ is an integral.

Let $E \subseteq I$. Let $f_{E}=f_{\chi_{E}}$, where $\chi_{E}$ is the characteristic function of $E$. One says $f$ is $T$-integrable on $E$ if $f_{E}$ is $T$-integrable on $I$.

One defines $\operatorname{dom}_{I} T^{H}$ by the following conditions:

$\left(\mathrm{h}_{1}\right) f$ is $T$-integrable on $\mathscr{S}$ and on each of the intervals $I_{k}$ contiguous to $\mathscr{S} \cup\{a, b\}$.

$\left(\mathrm{h}_{2}\right) \sum O\left(T ; f ; I_{k}\right)<\infty$, where $O(T ; f ; J)=\sup _{J^{\prime} \subseteq J}\left|T\left(f, J^{\prime}\right)\right|$.

Define $T^{H}(f, I)=T(f, \mathscr{S})+\sum T\left(f, I_{k}\right)$. Clearly $T^{H}$ is also an integral.

Let $\left\{T^{\alpha}\right\}$ be a sequence of integrals, in general transfinite, such that $T^{\alpha} \subseteq T^{\beta}$ whenever $\alpha<\beta$. Define dom $\sum_{\beta<\alpha} T^{\beta}=\bigcup_{\beta<\alpha} \operatorname{dom} T^{\beta}$ and if $\left(f, I^{\prime}\right) \in \operatorname{dom} \sum_{\beta<\alpha} T^{\beta}$, define $\left(\sum_{\beta<\alpha} T^{\beta}\right)\left(f, I^{\prime}\right)=T^{\beta_{0}}\left(f, I^{\prime}\right)$, where $\beta_{0}$ is the least ordinal such that $\left(f, I^{\prime}\right) \in \operatorname{dom} T^{\beta_{0}}$.

Write $T^{C H}=\left(T^{C}\right)^{H}$. We define a transfinite sequence $\left\{D^{\alpha}\right\}$ of integrals as follows: let $\mathscr{L}$ be the Lebesgue integral,

$$
\begin{aligned}
& D^{0}=\mathscr{L}, \\
& D^{\alpha}=\left(\sum_{\beta<\alpha} D^{\beta}\right)^{C H}
\end{aligned}
$$

if $\alpha>0$. Let $\Omega$ be the first uncountable ordinal. Then it is well known ([2], 258) that if $D_{*}$ is the restricted Denjoy integral,

$$
D_{*}=\sum_{\alpha<\Omega} D^{\alpha} .
$$

2. A constructive definition using the Burkill integral. We define $\operatorname{dom}_{I} T^{H^{*}}$ by the following conditions:

(h\%) there exists a closed set $W \subseteq I$ such that $f$ is $T$-integrable on $W$ and on each $I^{\prime} \subseteq I$ with $I^{\prime} \cap W=\varnothing$;

$\left(\mathrm{h}_{2}^{*}\right)$ if we define

$$
\begin{aligned}
\psi\left(I^{\prime}\right) & =T\left(f, I^{\prime}\right) & & \text { if } I^{\prime} \cap W=\varnothing, \\
& =0 & & \text { if } I^{\prime} \cap W \neq \varnothing,
\end{aligned}
$$

then $\int_{I} \psi$ exists and $\int \psi$ is continuous (note that if $\int_{I} F$ exists, then $\int_{I^{\prime}} F$ exists for all $I^{\prime} \subseteq I$ and $\int_{I^{\prime}} F$ is additive).

Define $T^{H^{*}}(f, I)=T\left(f_{\mathrm{W}}, I\right)+\int_{I} \psi$. We note that $T^{H^{\bullet}}$ is an integral. For suppose that $f \in \operatorname{dom}_{J} T^{H^{*}}$. Let $J^{\prime} \subseteq J$. If $J^{\prime} \cap W=\varnothing$, then $f \in \operatorname{dom}_{J^{\prime}} T$ so that, taking $W^{\prime}=J^{\prime}$ in the definition of $\operatorname{dom}_{J^{\prime}} T^{H^{*}}$, we see that $f \in \operatorname{dom}_{J^{\prime}} T^{H^{*}}$. If $J^{\prime} \cap W \neq \varnothing$, then since $f_{\mathrm{W}} \in \operatorname{dom}_{J} T$ and $T$ is an integral, $f_{\mathrm{W}} \in \operatorname{dom}_{J}, T$. Now, since $\int_{J} \psi$ exists, so does $\int_{J^{\prime}} \psi([1]$, p. 70$)$. Recall that continuity of $\int \psi$ is assumed in the definition 
of $\operatorname{dom}_{J} T^{H^{*}}$. Therefore, in either case $f \in \operatorname{dom}_{J^{\prime}} T^{H^{*}}$. Since $T$ is an integral, $T\left(f_{\mathrm{W}}, J^{\prime}\right)$ is an additive, continuous function of the $J^{\prime} \subseteq J$; it is known ([1], p. 70) that $\int \psi$ is additive. Therefore $T^{H^{*}}\left(f, J^{\prime}\right)$ is an additive, continuous function of the $J^{\prime} \subseteq J$, and condition (i) is satisfied. Now suppose $f \in \operatorname{dom}_{I_{i}} T^{H^{*}}, i=1,2$, where $I_{1}$ and $I_{2}$ are abutting. With no loss in generality we may assume $I_{1}$ is to the left of $I_{2}$. Choose $W_{i} \subseteq I_{i}, i=1,2$, closed sets satisfying the requirements of the definition of $f \in \operatorname{dom}_{I_{i}} T^{H^{*}}$, and let $\psi_{i}$ be the functions corresponding to $T^{H^{*}}, f$ and $W_{i}$, $i=1,2$, in this definition. Set $a_{1}=\sup W_{1}, b_{1}=\inf W_{2}$. If $a_{1}=b_{1}$, choose

$$
W=W_{1} \cup W_{2} \text {. }
$$

Then $W$ is closed and, since $T$ is an integral, by condition (ii), $f_{W} \in \operatorname{dom}_{I_{1} \cup I_{2}} T$. Let $\varepsilon>0$ and choose $\eta_{i}=\eta_{i}(\varepsilon)>0, i=1,2$, so that if $P_{i}$ is a partition of $I_{i}, i=1,2$, with $\left|P_{i}\right|<\eta_{i}$, then $\left|\sum_{I^{\prime} \in P_{i}} \psi_{i}\left(I^{\prime}\right)-\int_{I_{i}} \psi_{i}\right|<\varepsilon / 8$, and also so that if $\left\{J_{1}, \ldots, J_{p}\right\}$ is any finite sequence of nonoverlapping subintervals of $I_{1}$ with $\operatorname{Max}\left|J_{k}\right|<\eta_{i}$, then $\sum_{1}^{p}\left|\psi_{i}\left(J_{k}\right)-\int_{J_{k}} \psi_{i}\right|<\varepsilon / 8$ (see [1], p. 70). Also, since $\int \psi_{i}$ is additive and continuous on $I_{k}, i=1,2$, we may choose $\eta, 0<\eta<\min \left(\eta_{1}, \eta_{2}\right)$, so that $J \subseteq I_{i},|J|<\eta$ imply that $\left|\int_{J} \psi_{i}\right|<\varepsilon / 8$. Now let $P$ be any partition of $I_{1} \cup I_{2}$ with $|P|<\eta$. Then

$$
\left|\sum_{I^{\prime} \in P} \psi\left(I^{\prime}\right)-\left(\int_{I_{1}} \psi_{1}+\int_{I_{2}} \psi_{2}\right)\right|<\varepsilon
$$

It therefore follows that $\int_{I_{1} \cup I_{2}} \psi=\int_{I_{1}} \psi_{1}+\int_{I_{2}} \psi_{2}$. It is immediate that $\int \psi$ is continuous on $I_{1} \cup I_{2}$. If $a_{1}<b_{1}$, choose $W=W_{1} \cup W_{2} \cup\left[a_{1}, b_{1}\right]$. A similar argument shows that, again, condition (ii) is satisfied. Finally, if $f \equiv 0$ on $I$, then since $T$ is an integral, $f \in \operatorname{dom}_{I} T$ and $T(f, I)=0$. Choosing $W=I$, we see that $f \in \operatorname{dom}_{I} T^{H^{*}}$ and $T^{H^{*}}(f, I)=0$. Therefore $T^{H^{*}}$ is an integral. By a known result ([1],90), if $T \subseteq D_{*}$, then $T^{H^{*}} \subseteq D_{*}$.

Romanovski ([1], 91) has defined the following sequence $\left\{K_{\alpha}\right\}$ of classes of $D_{*}$-integrable functions defined on $I$. Put $f \in K_{0}$ if $f$ is Lebesgue integrable on $I$. Let $\alpha>0$. Then we put $f \in K_{\alpha}$ if one of the following four conditions is satisfied:

(1) there is a partition $P$ such that $f$ is in a class $K_{\beta}$, with $\beta<\alpha$, on each member of $P$;

(2) $f$ can be extended to a function which belongs to a class $K_{\beta}$, with $\beta<\alpha$, on some $I^{\prime} \supseteq I$;

(3) $f$ belongs to a class $K_{\beta}$, with $\beta<\alpha$, on each closed sub-interval of $I^{0}=(a, b)$;

(4) there is a closed set $W$ such that $f_{\mathrm{W}}$ is Lebesgue integrable on $I$, and such that, on each $I^{\prime} \subseteq I$ with $I^{\prime} \cap W=\varnothing, f$ belongs to a class $K_{\beta}$, with $\beta<\alpha$; in addition, if

$$
\begin{aligned}
\psi\left(I^{\prime}\right) & =\left(D_{*}\right) \int_{I^{\prime}} f d x & & \text { if } I^{\prime} \cap W=\varnothing, \\
& =0 & & \text { if } I^{\prime} \cap W \neq \varnothing,
\end{aligned}
$$

then $\int_{I} \psi$ exists and $\int \psi$ is continuous. It is known $([1], 91)$ that if $f$ is $D_{*}$-integrable, then $f$ belongs to some class $K_{\alpha}$. 
We define a transfinite sequence $\left\{D_{*}^{\alpha}\right\}$ of integrals as follows:

if $\alpha>0$.

$$
\begin{aligned}
& D_{*}^{0}=\mathscr{L}, \\
& D_{*}^{\alpha}=\left(\sum_{\beta<\alpha} D_{*}^{\beta}\right)^{H^{*}}
\end{aligned}
$$

THEOREM 1. $K_{\alpha} \subseteq \operatorname{dom}_{I} D_{*}^{\alpha+1}$.

Proof. Clearly $\operatorname{dom}_{I} D_{*}^{0}=K_{0}$. Suppose the theorem is true for all $\beta<\alpha$, for some $\alpha>0$ and that $f \in K_{\alpha}$. If condition (1) of the definition of $K_{\alpha}$ is satisfied, then there is a partition $P$ of $I$ such that $f \in \operatorname{dom}_{I^{\prime}} D_{*}^{\beta+1}$ for all $I^{\prime} \in P$, where $\beta<\alpha$. By condition (ii) for an integral, $f \in \operatorname{dom}_{I} D_{*}^{\beta+1}$. Since $\beta+1<\alpha+1, f \in \operatorname{dom}_{I} D_{*}^{\alpha+1}$. If condition (2) of the definition of $K_{\alpha}$ is satisfied, then there exists $I^{\prime} \supseteq I$ such that $f \in K_{\beta}$ on $I^{\prime}$, $\beta<\alpha$. But then by the induction hypothesis, $f \in \operatorname{dom}_{I^{\prime}} D_{*}^{\beta+1}$ and by condition (i) for integrals, $f \in \operatorname{dom}_{I} D_{*}^{\beta+1}$. Since $\beta+1<\alpha+1$, again $f \in \operatorname{dom}_{I} D_{*}^{\alpha+1}$. Now suppose condition (4) of the definition of $K_{\alpha}$ is satisfied. Then there is a closed set $W \subseteq I$ such that $f_{W} \in \operatorname{dom}_{I} \mathscr{L}$, and such that, on each $I^{\prime} \subseteq I$ with $I^{\prime} \cap W=\varnothing$, $f \in \operatorname{dom}_{I^{\prime}} D_{*}^{\beta+1}$, where $\beta<\alpha$; in addition, $\int_{I} \psi$ exists and $\int \psi$ is continuous. It follows that $f \in \operatorname{dom}_{I} D_{*}^{\alpha+1}$.

We finally show that if condition (3) of the definition is satisfied, then $f$ is $D_{*}^{\alpha+1}$-integrable. Now $f \in \operatorname{dom}_{I^{\prime}} D_{*}^{\beta}$, with $\beta<\alpha+1$, for all $I^{\prime} \subseteq(a, b)$. Let

$$
F=\left(D_{*}\right) \int f d x
$$

Then $F$ is continuous on $I$. In $\left(\mathrm{h}_{1}^{*}\right)$ and $\left(\mathrm{h}_{2}^{*}\right)$ choose $T=D_{*}^{\alpha}$. Note that $F\left(I^{\prime}\right)=T\left(f, I^{\prime}\right)$ for all $I^{\prime} \subseteq I^{0}$. Let $W=\{a, b\}$ and $\varepsilon>0$. Choose $\delta>0$ such that $I^{\prime} \subseteq I,\left|I-I^{\prime}\right|<\delta$ imply $\left|F(I)-F\left(I^{\prime}\right)\right|<\varepsilon$. Let $P$ be any partition of $I$ with $|P|<\delta / 2$. Then

$$
\left|\sum_{I^{\prime} \in P} \psi\left(I^{\prime}\right)-F(I)\right|=\left|\sum_{I^{\prime} \in P^{\prime}} F\left(I^{\prime}\right)-F(I)\right|<\varepsilon,
$$

where $P^{\prime}$ is the set of those members of $P$ which contain neither $a$ nor $b$. It follows that $\int_{I} \psi=F(I)$. Clearly $\int \psi$ is continuous. Therefore

$$
K_{\alpha} \subseteq \operatorname{dom} D_{*}^{\alpha+1}
$$

THEOREM 2. $D_{*}=\sum_{\alpha<\Omega} D_{*}^{\alpha}$.

Proof. Let $f$ be $D_{*}$-integrable on $I$. Define $f=0$ outside of $I$. Let $S^{\alpha}$ denote the set of $D_{*}^{\alpha}$-singular points of $f$ in $I$. Then $\left\{S^{\alpha}\right\}$ is decreasing and, thus, stationary (i.e., there is an $\alpha_{0}<\Omega$ such that $S^{\alpha}=S^{\alpha}$ for all $\alpha>\alpha_{0}$; see, e.g., [2], 258). However, $f \in K_{\beta}$ for some $\beta$ and, therefore, $f \in \operatorname{dom}_{I} D_{*}^{\beta+1}$. Thus $S^{\beta+1}=\varnothing$, which implies $S^{\alpha_{0}}=\varnothing$ and $f \in \operatorname{dom}_{I} D_{*}^{\alpha_{0}}$. We have observed above that $T \subseteq D_{*}$ implies $T^{H^{*}} \subseteq D_{*}$. To show that $D_{*} \supseteq \sum_{\alpha<\Omega} D_{*}^{\beta}$ it is easy to see that if $D_{*}^{\beta} \subseteq D_{*}$ for all $\beta<\alpha$, then $\sum_{\beta<\alpha} D_{*}^{\beta} \subseteq D_{*}$, and that therefore $\left(\sum_{\beta<\alpha} D_{*}^{\beta}\right)^{H^{*}} \subseteq D_{*}$. Since $D_{*}^{0}=\mathscr{L} \subseteq D_{*}$, we have the desired containment relation by applying transfinite induction. 
3. Comparison of our construction with the classical construction. In the following theorem we restrict our attention to those integrals $T$ which have the property that $f \in \operatorname{dom}_{I} T$ whenever $I \cap\{f \neq 0\}$ is finite.

THEOREM 3. $T^{C} \subseteq T^{H^{*}}$.

Proof. Let $f$ be $T^{C}$-integrable on $I$. Let $\mathscr{S}$ be the set of $T$-singular points in $I$ and $\left\{I_{1}, \ldots, I_{k}\right\}$ be the set of intervals contiguous to $\mathscr{S} \cup\{a, b\}=E$. Let $F\left(I^{\prime}\right)=T^{C}\left(f, I^{\prime}\right)$ for all $I^{\prime} \subseteq I$. Let $\varepsilon>0$. Choose $\delta>0$ such that for each $i, I^{\prime} \subseteq I_{i}$ and $\left|I_{i}-I^{\prime}\right|<\delta$ imply $\left|F\left(I_{i}\right)-F\left(I^{\prime}\right)\right|<\varepsilon / k$. Let $S_{I}=\left\{J_{1}, \ldots, J_{m}\right\}$ be any partition of $I$ with $\left|S_{I}\right|<\delta / 2$. Define

$$
\begin{aligned}
\psi\left(I^{\prime}\right) & =F\left(I^{\prime}\right) & & \text { if } I^{\prime} \cap E=\varnothing, \\
& =0 & & \text { if } I^{\prime} \cap E \neq \varnothing,
\end{aligned}
$$

for all $I^{\prime} \subseteq I$. If $S \subseteq S_{I}$ is the subset of $S_{I}$ consisting of those $J_{i}$ such that $J_{i} \cap E=\varnothing$, we see that, since $F$ is additive,

$$
\left|\sum_{i=1}^{m} \psi\left(J_{i}\right)-F(I)\right|=\left|\sum_{J_{i} \in S} \psi\left(J_{i}\right)-\sum F\left(I_{j}\right)\right|<\varepsilon .
$$

It follows that $\int_{I} \psi=F(I)$.

We shall show below that the containment guaranteed by Theorem 3 is proper in general, i.e., there are $T^{H^{*}}$-integrable functions which are not $T^{C}$-integrable.

We note that $\left(\mathrm{h}_{1}^{*}\right)$ and $\left(\mathrm{h}_{2}^{*}\right)$ do not require that $f$ be $T$-integrable on the intervals $I_{j}$ contiguous to $W$. Since $\int_{I} \psi$ exists, $\int \psi$ is continuous, and $T\left(f, I^{\prime}\right)$ is an additive function of the closed subintervals of $I_{j}^{0}$ for all $j$, it follows that $\int_{I^{\prime}} \psi=T\left(f, I^{\prime}\right)$ for all such $I^{\prime}$, and therefore $f$ is $T^{C}$-integrable on $I_{j}$ for all $j$. Moreover,

$$
\int_{I_{j}} \psi=\lim _{\left|I_{j}-I^{\prime}\right| \rightarrow 0} T\left(f, I^{\prime}\right) .
$$

The following theorem will immediately establish the inclusion relationships between $T^{H}, T^{H^{*}}$, and $T^{C H}$.

THEOREM 4. Suppose $E \subseteq I$ is closed. Let $\left\{I_{k}\right\}$ be the sequence of intervals contiguous to $E \cup\{a, b\}$. Consider the following two conditions:

(1) for every $k, f \in \operatorname{dom}_{I^{\prime}} T$ for all $I^{\prime} \subseteq I_{k}^{0}$ and $f \in \operatorname{dom}_{I_{k}} T^{C}$; moreover, $\sum O\left(T^{C} ; f ; I_{k}\right)<\infty$;

(2) $f$ is $T$-integrable on all $I^{\prime} \subseteq I$ such that $I^{\prime} \cap E=\varnothing$; moreover, if

$$
\begin{aligned}
\psi\left(I^{\prime}\right) & =T\left(f, I^{\prime}\right) & & \text { if } I^{\prime} \cap E=\varnothing, \\
& =0 & & \text { if } I^{\prime} \cap E \neq \varnothing,
\end{aligned}
$$

then $\int_{I} \psi$ exists and $\int \psi$ is continuous. Then (1) and (2) are equivalent.

Corollary 5. $T^{H} \subseteq T^{H^{*}}$.

COROLlaRY 6. $T^{H^{*}} \subseteq T^{C H}$. 
These corollaries follow from a comparison of the conditions in the definitions of $T^{H}, T^{H^{*}}$, and $T^{C H}$ with conditions (1) and (2) of Theorem 4.

Proof of Theorem 4. We show first that (1) implies (2). Let $\varepsilon>0$. Choose $N=N(\varepsilon)$ such that

$$
\sum_{N+1}^{\infty} O\left(T^{c} ; f ; I_{k}\right)<\varepsilon / 3 .
$$

Let $I_{k}=\left[a_{k}, b_{k}\right]$ for all $k$. Consider $I_{j}$ with $j \leqq N$. By continuity of $T^{C}\left(f, I^{\prime}\right)$ there is a $\delta_{j}=\delta_{j}(\varepsilon)>0$ such that for $I^{\prime} \subseteq I_{j},\left|T^{C}\left(f, I_{j}\right)-T^{C}\left(f, I^{\prime}\right)\right|<\varepsilon / 6 N$ whenever $\left|I_{j}-I^{\prime}\right|<\delta_{j}$. In particular, then, if $J^{\prime}=\left[a_{j}, b^{\prime}\right]$ and $J^{\prime \prime}=\left[a^{\prime \prime}, b_{j}\right]$ are contained in $J_{j}$, and if $b^{\prime}-a_{j}<\delta_{j}$ and $b_{j}-a^{\prime \prime}<\delta_{j}$, then

$$
\left|T^{C}\left(f, J^{\prime}\right)\right|+\left|T^{C}\left(f, J^{\prime \prime}\right)\right|<\varepsilon / 3 N .
$$

Note, also, that since $T^{C}(f, I)$ is additive, if $J^{\prime} \subseteq I_{j}$ and $\left\{J_{1}^{\prime}, \ldots, J_{p}^{\prime}\right\}$ is a partition of $J^{\prime}$, then $\sum T^{C}\left(f, J_{i}^{\prime}\right)=T^{C}\left(f, J^{\prime}\right)$.

Now choose $\delta=\min _{j=1, \ldots, N} \delta_{j}$. Suppose $S=\left\{J_{1}, \ldots, J_{p}\right\}$ is a partition of $I$ with $|S|<\delta$. The only members $J_{i}$ of $S$ for which it is possible that $\psi\left(J_{\imath}\right) \neq 0$ are those for which $J_{i} \cap E=\varnothing$. Consider $I_{k}$ with $k \leqq N$. Let $\left\{J_{1}^{k}, \ldots, J_{n(k)}^{k}\right\}$ be those $J_{i}$ which are contained in $I_{k}$, such that $J_{m}^{k} \cap E=\varnothing$. Then by the above discussion we see that

$$
\left|\sum_{m=1}^{n(k)} \psi\left(J_{m}^{k}\right)-T^{C}\left(f, I_{k}\right)\right|<\varepsilon / 3 N .
$$

For $k>N$, clearly $\left|\sum_{m=1}^{n(k)} \psi\left(J_{m}^{k}\right)\right| \leqq O\left(T^{C} ; f ; I_{k}\right)$. Thus,

$$
\begin{aligned}
\left|\sum_{k=1}^{p} \psi\left(J_{k}\right)-\sum_{k=1}^{\infty} T^{C}\left(f, I_{k}\right)\right| \\
=\left|\sum_{k=1}^{\infty} \sum_{m=1}^{n(k)} \psi\left(J_{m}^{k}\right)-\sum_{k=1}^{N} T^{C}\left(f, I_{k}\right)-\sum_{k=N+1}^{\infty} T^{C}\left(f, I_{k}\right)\right| \\
\quad \leqq\left|\sum_{k=1}^{N}\left[\sum_{m=1}^{n(k)} \psi\left(J_{m}^{k}\right)-T^{C}\left(f, I_{k}\right)\right]\right|+\sum_{k=N+1}^{\infty}\left|\sum_{m=1}^{n(k)} \psi\left(J_{m}^{k}\right)\right|+\sum_{k=N+1}^{\infty}\left|T^{C}\left(f, I_{k}\right)\right| \\
\quad<N_{\varepsilon} / 3 N+2 \sum_{N+1}^{\infty} O\left(T^{C} ; f ; I_{k}\right)<\varepsilon .
\end{aligned}
$$

Thus $\int_{I} \psi$ exists and $\int_{I} \psi=\sum_{1}^{\infty} T^{C}\left(f, I_{k}\right)$. The continuity of $\int \psi$ is clear.

We now show that (2) implies (1). Let $\delta=\delta(1)>0$ be such that

$$
\left|\sum_{I^{\prime} \in S_{I}} \psi\left(I^{\prime}\right)-\int_{I} \psi\right|<1
$$

whenever $S_{I}$ is a partition of $I$ with $\left|S_{I}\right|<\delta$, and also such that

$$
\left|\sum_{I^{\prime} \in S} \psi\left(I^{\prime}\right)-\sum_{I^{\prime} \in S} \int_{I^{\prime}} \psi\right|<1
$$

whenever $S$ is a finite family of nonoverlapping subintervals of $I$ with $\sup _{I^{\prime} \in S}\left|I^{\prime}\right|<\delta$ (see [1], 70). 
Choose $N=N(\delta)$ such that $\sum_{N+1}^{\infty}\left|I_{k}\right|<\delta / 2$. We shall divide $\left\{I_{k}\right\}_{N+1}^{\infty}$ into two classes, $\mathscr{I}^{+}$and $\mathscr{I}^{-}$, as follows: put $I_{k}$ in $\mathscr{I}^{+}$if there exists $\left\{I_{j}^{k}\right\}$, with $I_{j}^{k} \subseteq I_{k}$, such that $T^{C}\left(f, I_{j}^{k}\right) \rightarrow O\left(T^{C} ; f ; I_{k}\right)$ and put $I_{k}$ in $\mathscr{I}^{-}$otherwise. Write $\mathscr{I}^{+}=\left\{I_{n_{i}}\right\}$ $=\left\{J_{i}\right\}$. Choose $J_{i}^{\prime}$ with $J_{i}^{\prime} \subseteq J_{i}^{0}$, such that $O\left(T^{c} ; f ; J_{i}\right)-T^{c}\left(f, J_{i}^{\prime}\right)<1 / 2^{i}$. We shall show that $\sum O\left(T^{C} ; f ; J_{i}\right)<\infty$. A similar argument yields the same inequality for the sum of the oscillations over the members of $\mathscr{I}^{-}$. The choice of $J_{i}^{\prime}$ induces a partition of $J_{i},\left\{J_{i}^{\prime}, J_{i}^{\prime \prime}, J_{i}^{\prime \prime}\right\}$. Append to the family $\left\{J_{i}\right\}$ the family $\left\{I_{1}, \ldots, I_{n}\right\}$. For each $k=1, \ldots, N$, choose a partition $\left\{I_{1}^{k}, \ldots, I_{n(k)}^{k}\right\}$ (enumerated from left to right) of $I_{k}$ such that $\left|I_{j}^{k}\right|<\delta$ for all $j$ and such that

$$
\left|T^{C}\left(f, I_{k}\right)-\sum_{2}^{n(k)-1} T^{C}\left(f, I_{j}^{k}\right)\right|<1 / 2 N .
$$

This can be done since $T^{C}(f, I)$ is additive and continuous on $I_{k}$ for all $k$. For any positive integer $p$, consider

$$
H=I-\left[\left(\bigcup_{1}^{N} I_{j}\right) \cup\left(\bigcup_{1}^{p} J_{i}\right)\right] .
$$

If $H$ is nonvoid, it consists of a finite number of intervals. We shall now form a partition $S$ of $I$. Put $\left\{J_{i}^{\prime}, J_{i}^{\prime \prime}, J_{i}^{m}\right\}_{i=1}^{p} \cup\left\{I_{1}^{k}, \ldots, I_{n(k)}^{k}\right\}_{k=1}^{N}$ into $S$. Suppose $I^{\prime}=\left[a^{\prime}, b^{\prime}\right]$ and $I^{\prime \prime}=\left[a^{\prime \prime}, b^{\prime \prime}\right]$ are two of these intervals with $b^{\prime}<a^{\prime \prime}$ such that none of the above intervals intersects $\left(b^{\prime}, a^{\prime \prime}\right)$. Then, since the sum of the lengths of the contiguous intervals contained in $H$ is $<\delta / 2$, it follows that we can partition $\left(b^{\prime}, a^{\prime \prime}\right)$ by a sequence of intervals all of whose end-points are in $E$, and all of which have length $<\delta / 2$. We put this partition into $S$. We do this for all pairs $I^{\prime}$ and $I^{\prime \prime}$ satisfying the above conditions. We thereby obtain a partition $S$ of $I$ such that $|S|<\delta$. Then

$$
\left|\int_{I} \psi-\sum_{I^{\prime} \in S} \psi\left(I^{\prime}\right)\right|<1
$$

But

Thus

$$
\sum_{I^{\prime} \in S} \psi\left(I^{\prime}\right)=\sum_{j=1}^{N} \sum_{k=2}^{n(j)-1} T\left(f, I_{k}^{j}\right)+\sum_{i=1}^{p} T\left(f, J_{i}^{\prime}\right) .
$$

$$
\begin{aligned}
& \sum_{i=1}^{p} T\left(f, J_{j}^{\prime}\right) \leqq\left|\sum_{j=1}^{N} \sum_{k=2}^{n(j)-1} T\left(f, I_{k}^{j}\right)\right|+1+\left|\int_{I} \psi\right| \\
& \leqq 1+\left|\int_{I} \psi\right|+N / 2 N+\sum_{1}^{N}\left|T^{C}\left(f, I_{k}\right)\right|<2+\left|\int_{I} \psi\right|+\sum_{1}^{N}\left|T^{C}\left(f, I_{k}\right)\right| .
\end{aligned}
$$

Since $O\left(T^{c} ; f ; J_{i}\right)-T\left(f, J_{i}^{\prime}\right)<1 / 2^{i}$ for all $i$,

$$
\begin{aligned}
\sum_{i=1}^{p} O\left(T^{c} ; f ; J_{i}\right) & \leqq \sum_{i=1}^{p} 1 / 2^{i}+2+\left|\int_{I} \psi\right|+\sum_{1}^{N}\left|T^{C}\left(f, I_{k}\right)\right| \\
& <3+\left|\int_{I} \psi\right|+\sum_{1}^{N}\left|T^{C}\left(f, I_{k}\right)\right| .
\end{aligned}
$$


Since $3+\left|\int_{I} \psi\right|+\sum_{1}^{N}\left|T^{C}\left(f, I_{k}\right)\right|$ is an absolute finite constant and $p$ was arbitrary, $\sum_{1}^{\infty} O\left(T^{c} ; f ; J_{i}\right)<\infty$. As we noted above, a similar argument can be used to establish the result for $\mathscr{I}^{-}$. Thus (2) implies (1).

The following example illustrates the fact that

$$
T^{H} \varsubsetneqq T^{H^{*}} \text { and } T^{C} \varsubsetneqq T^{H^{*}} \text {. }
$$

Let

$$
\left\{I_{n}\right\}=\left\{\left[\frac{1}{n+1}, \frac{1}{n}\right]\right\} n=1,2, \ldots
$$

Define

$$
\begin{array}{rlrl}
f(x) & =x^{2} \sin \frac{1}{x^{2}} & & \text { if } 0<x \leqq \frac{1}{2}, \\
& =\left(\frac{1}{2} \sin 4\right)(1-x) & \text { if } \frac{1}{2} \leqq x \leqq 1 .
\end{array}
$$

Extend $f$ by the formula

$$
f(x+n)=f(x)
$$

for all integers $n$. Define

$$
\begin{aligned}
f_{n}(x) & =\frac{1}{2^{n+1}} f(n(n+1) x) & & \text { if } x \in I_{n}, \\
& =0 & & \text { if } x \notin I_{n},
\end{aligned}
$$

and

$$
F(x)=\sum_{1}^{\infty} f_{n}(x)
$$

for all $x \in I$. $F(x)$ is clearly continuous on $I$ and differentiable a.e. Let $g(x)=F^{\prime}(x)$. Let $E=\{0\} \cup\{1 / n\}, n=2,3, \ldots$ Clearly $g$ is $\mathscr{L}^{C}$-integrable on $I_{n}$ for all $n$. By Theo-

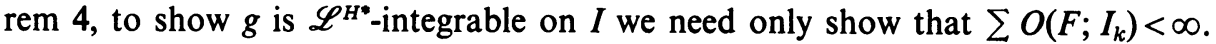
Since $O\left(F ; I_{k}\right)<1 / 2^{k}$, this is immediate. Thus $g$ is $\mathscr{L}^{H^{*}}$-integrable. However, since $E$ is the set of $\mathscr{L}$-singular points of $g$, and since $g$ is not $\mathscr{L}$-integrable on $I_{n}$ for any $n$, it follows that $g$ is not $\mathscr{L}^{H}$-integrable. It is clear that $g$ is not $\mathscr{L}^{\mathrm{C}}$-integrable.

Finally we shall show that $T^{H^{*}} \varsubsetneqq T^{C H}$. Let $E$ be the Cantor set. Let $\left\{I_{n}\right\}=\left\{\left[a_{n}, b_{n}\right]\right\}$ be the sequence of intervals contiguous to $E$. Let $f(x)$ be as in the previous example and

$$
\begin{aligned}
F_{n}(x) & =\frac{1}{2^{n+1}} f\left(\frac{2^{n+1}\left(x-a_{n}\right)}{\left|I_{n}\right|}\right) & & \text { if } x \in I_{n} \\
& =0 & & \text { otherwise }
\end{aligned}
$$

$n=1,2, \ldots$ Define

$$
F(x)=\sum F_{n}(x)
$$


Clearly $g(x)=F^{\prime}(x)$ exists a.e. Moreover, each point of $E$ is an $\mathscr{L}^{c}$-singular point of $g$, and no other point is singular. In addition, $O\left(F ; I_{n}\right)<1 / 2^{n}$. Thus $\sum O\left(F ; I_{n}\right)$ $<\infty$, implying that $g$ is $\mathscr{L}^{C H}$-integrable. However, $I_{n}$ contains $2^{n+1}+1 \mathscr{L}$-singular points, namely $\left\{a_{n}+j\left(\left|I_{n}\right| / 2^{n+1}\right)\right\} j=0,1, \ldots, 2^{n+1}$. In addition,

$$
O\left(F ;\left[a_{n}+j \frac{\left|I_{n}\right|}{2^{n+1}}, a_{n}+(j+1) \frac{\left|I_{n}\right|}{2^{n+1}}\right]\right)=\frac{1}{2^{n+1}} O(f ;[0,1])
$$

for all $j$ and $n$. Then, if $H=E \cup\left[\bigcup_{n=1}^{\infty} \bigcup_{j=0}^{2^{n+1}}\left\{a_{n}+j\left(\left|I_{n}\right| / 2^{n+1}\right)\right\}\right]$, we note that $H$ is closed and that $\sum O\left(F ; J_{k}\right)=\infty$, where $\left\{J_{k}\right\}$ is the sequence of intervals contiguous to $H$. Moreover, if $P \subseteq I$ is a closed set such that $g$ is $\mathscr{L}$-integrable on $P$ and on all $I^{\prime} \subseteq I$ with $I^{\prime} \cap P=\varnothing$, then $\sum O\left(F ; I_{j}^{\prime}\right)=\infty$, where $\left\{I_{j}^{\prime}\right\}$ is the sequence of intervals contiguous to $P$. For, let

$$
I_{n j}=\left[a_{n}+j\left(\left|I_{n}\right| / 2^{n+1}\right), a_{n}+(j+1)\left(\left|I_{n}\right| / 2^{n+1}\right)\right],
$$

$j=0,1, \ldots, 2^{n+1}-1$, and write $a_{n j}=a_{n}+j\left(\left|I_{n}\right| / 2^{n+1}\right)$. Let $c_{n j}$ be the midpoint of $I_{n j}$. Note that $F$ is monotone increasing on $\left[c_{n j}, a_{n, j+1}\right]$ and that $g$ is positive on $\left[c_{n j}, a_{n, j+1}\right]$. Let $\left\{I_{n j}^{k}\right\}$ be the sequence of intervals contiguous to $P \cup\left\{c_{n j}, a_{n, j+1}\right\}$ in $\left[c_{n j}, a_{n, j+1}\right]$. Then either

$$
\int_{\left.P \cap\left[c_{n}\right\}, a_{n, j+1}\right]} g d x \geqq \frac{1}{8}|\sin 4|
$$

or

$$
\sum_{k} O\left(F ; I_{n j}^{k}\right) \geqq \frac{1}{8}|\sin 4|
$$

If

$$
\int_{P \cap\left[c_{n}, a_{n, j+1}\right]} g d x \geqq \frac{1}{8}|\sin 4|
$$

for infinitely many pairs $n, j$, then $\int_{P}|g| d x=\infty$ contradicting the assumption that $g$ is $\mathscr{L}$-integrable on $P$. Thus, assuming that $g$ is $\mathscr{L}$-integrable on $P$,

$$
\sum_{k} O\left(F ; I_{n j}^{k}\right) \geqq|\sin 4| / 8
$$

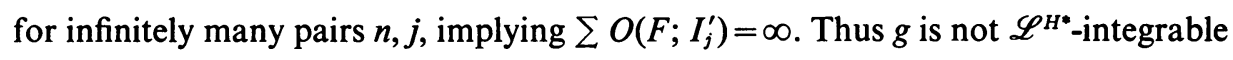
although it is $\mathscr{L}^{\mathrm{CH}}$-integrable.

\section{REFERENCES}

1. P. Romanovski, Intégrale de Denjoy dans les espaces abstraits, Mat. Sb. 9(51) (1941), 67-120.

2. S. Saks, Theory of the integral, Hafner, New York, 1937.

UNIVERSITY OF WISCONSIN, MilWAUKeE, Wisconsin 